\title{
TEM Investigation of Nanocarriers Distribution in Mice Brain
}

\author{
Roozbeh Nikkhah-Moshaie ${ }^{1}$, Ajeet Kaushik ${ }^{2}$, Rahul D. Jayant ${ }^{2}$, Vinay Bhardwaj ${ }^{3}$, Madhavan Nair ${ }^{2}$ \\ ${ }^{1 .}$ Department of Mechanical \& Materials Engineering, College of Engineering and Computing, Florida \\ International University, Miami, USA \\ 2. Center for Personalized Nanomedicine, Institute of Neuroimmune Pharmacology, Department of \\ Immunology, Herbert Wertheim College of Medicine, Florida International University, Miami, USA \\ ${ }^{3 .}$ Department of Chemistry and Physics, Western Carolina University, Cullowhee, USA
}

Scanning-Transmission Electron Microscopy (STEM) is a useful technique to explore nanosize features in materials science, biology, and nanomedicine. Combination of STEM imaging, chemical analysis by EDS, and electron diffraction techniques provides a unique opportunity to investigate nanocarriers in drug delivery. Brain is a target for many diseases including tumors, Alzheimer, and neuro-AIDS [1]. Metal and oxide nanoparticles including iron \& magnetite [2,3] and barium titanate \& cobalt ferrite [4] are potential nanocarriers for drug delivery and sensing applications.

The phase composition of synthesized nanoparticles was studied using an X-ray diffractometer with Mo$\mathrm{K} \alpha$ radiation. Injection of magneto-electric nanocarriers (MENCs) of $\mathrm{BaTiO}_{3} @ \mathrm{CoF}_{2} \mathrm{O}_{4}$ were performed under a static magnetic field of $0.8 \mathrm{~T}$ for 3 hours. In order for TEM sample preparation, the ultrathin sections of mice brain were prepared using an ultramicrotome (Porter-Blum MT-1, DuPont-Sorvall, USA). The details of this procedure are given elsewhere [5]. The sections were loaded onto Ni grids for imaging experiments. FEI CM 200 and FEI Technai F30 were used to investigate the morphology of brain samples before and after the injection of MENCs. STEM was also used to study the particle size and distribution along with convergent beam electron diffraction (CBED) to determine crystalline characteristics of MENCs after injection.

Fig. 1 a exhibits the XRD pattern of MENCs, and is indexed according to $\mathrm{CoF}_{2} \mathrm{O}_{4}$ and $\mathrm{BaTiO}_{3}$ crystallographic planes. Comparing Fig $1 \mathrm{~b}$ and c shows that nanoparticles are inside the cells (Fig. $1 \mathrm{~b}$ before injection vs Fig. $1 \mathrm{c}$ after injection). Results of TEM micrographs confirmed that nanoparticles were uniformly distributed in all cell with particle size of 20 to $30 \mathrm{~nm}$. Fig. $1 \mathrm{~d}$ and e also shows the STEM images of the mice brain confirming the presence of nanoparticles inside the brain cells. Convergent beam electron diffraction of the nanoparticles confirms the presence of $\mathrm{BaTiO}_{3}$ and $\mathrm{CoF}_{2} \mathrm{O}_{4}$. (Fig. $1 \mathrm{f}$ ). Diffraction pattern results indicate that nanoparticles did not disintegrate during the complex process of delivery under magnetic field through blood and also during their presence inside brain cells.

In conclusion, the delivery of ferromagnetic nanocarriers under a static magnetic field to the brain of mice has been successfully demonstrated in this work. The results of TEM image analysis confirmed that nanocarriers not only navigated to the brain cells but also distributed throughout the brain even to the nuclei of cells. The diffraction study of nanoparticles in brain tissue confirmed that nanoparticles maintained their crystalline structure [6].

\section{References:}

[1] A. Kaushik et al, Exp. Opin. Drug Del. 11 (2014), p. 1635.

[2] A. Ataie and R. Nikkhah-Moshaie, Adv. Mater, Res. 55-57 (2008), p. 569. 
[3] A. Ataie et al, IEEE Trans. Magn. 45 (2009), p. 2496.

[4] M. Nair et al, Nat. Commun. 4 (2013), 1707.

[5] V. Bhardwaj et al, Analyst 140 (2015), p.3929.

[6] Authors acknowledge NIH grants namely RO1-DA027049, R21-MH 101025, RO1-DA 034547, R01-DA037838, RO1-DA-036154, and 1R01-DA-040537-01.
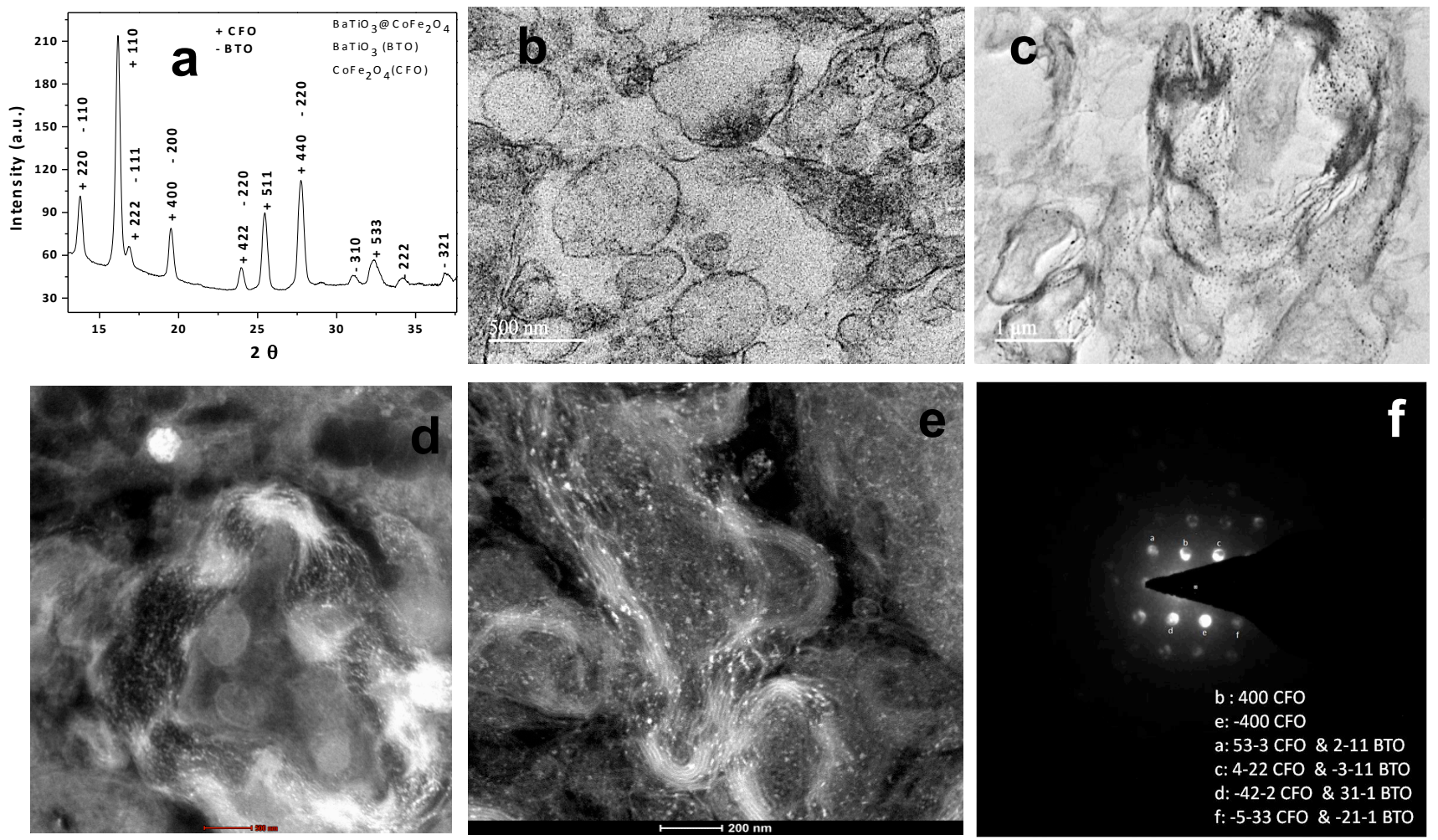

Figure 1. XRD pattern of nanoparticles (a), TEM image of mice brain tissue before injection (b) and after injection of nanoparticles (c), STEM images of mice brain tissue after injection (d and e), convergent beam electron diffraction pattern of nanoparticles (f). 